\title{
A fractional Fokker-Planck model for anomalous diffusion
}

\author{
Johan Anderson, ${ }^{1, a)}$ Eun-jin Kim, ${ }^{2}$ and Sara Moradi ${ }^{3}$ \\ ${ }^{1}$ Department of Earth and Space Sciences, Chalmers University of Technology, SE-412 96 Göteborg, Sweden \\ ${ }^{2}$ Department of Mathematics and Statistics, University of Sheffield, Hicks Building, Hounsfield Road, \\ Sheffield S3 7RH, United Kingdom \\ ${ }^{3}$ Ecole Polytechnique, CNRS UMR7648, LPP, F-91128 Palaiseau, France
}

(Received 16 September 2014; accepted 28 November 2014; published online 16 December 2014)

In this paper, we present a study of anomalous diffusion using a Fokker-Planck description with fractional velocity derivatives. The distribution functions are found using numerical means for varying degree of fractionality of the stable Lévy distribution. The statistical properties of the distribution functions are assessed by a generalized normalized expectation measure and entropy in terms of Tsallis statistical mechanics. We find that the ratio of the generalized entropy and expectation is increasing with decreasing fractionality towards the well known so-called sub-diffusive domain, indicating a self-organising behavior. [http://dx.doi.org/10.1063/1.4904201]

\section{INTRODUCTION}

In the early 20th century, Einstein studied classical diffusion in terms of Brownian motion. In this process, the mean value of the process vanishes, whereas the second moment or variance grows linearly with time $\left\langle\delta x^{2}\right\rangle=2 D t$. Anomalous diffusion, however, is in contrast to classical diffusion in terms of the variance that exhibit a non-linear increase with time $\left\langle\delta x^{2}\right\rangle=2 D t^{\alpha}$. There is no mechanism that inherently constrains $\lim _{\delta x, \delta t \rightarrow 0} \frac{\delta x^{2}}{\delta t}$ to be finite or non-zero. In more general terms, there are two limits of interest where the first is super-diffusion $\alpha>1$ and the second is sub-diffusion with $\alpha<1$. Such strange kinetics ${ }^{1-6}$ may be generated by accelerated or sticky motions along the trajectory of the random walk. $^{7}$ The main cause of anomalous super-diffusion is the existence of long-range correlations in the dynamics generated by the presence of anomalously large particle displacements.

Anomalous sub-diffusive properties have been studied in many different contexts, among them are that of holes in amorphous semiconductors where a waiting time distribution with long tails was introduced ${ }^{8}$ and the sub-diffusive processes within a single protein molecule described by generalized Langevin equation with fractional Gaussian noise. ${ }^{9}$ Moreover, it has been recognized that the nature of the transport processes common to plasma physics is dominated by turbulence with a significant ballistic or non-local component, where a diffusive description is improper. The basic mechanism underlying plasma transport is a very complex process and not very well understood. Furthermore, in plasma physics, super-diffusive properties are often found with $\alpha>1$ such as the thermal and particle flux in magnetically confined plasmas or transport in Scrape-Off Layer (SOL) dominated by coherent structures. ${ }^{10-14}$ In this paper, we will mainly concern ourselves with super-diffusion modeled by a Fractional Fokker-Planck equation (FFPE).

A salient component describing the suggestive non-local features of plasma turbulence is the inclusion of a fractional velocity derivative in the Fokker-Planck (FP) equation leading to an inherently non-local description as well as giving

\footnotetext{
${ }^{\text {a)} E l e c t r o n i c ~ m a i l: ~ a n d e r s o n . j o h a n @ g m a i l . c o m ~}$
}

rise to non-Gaussian probability density functions (PDFs) of, e.g., densities and heat flux. Note that the non-Gaussian features of the PDFs heat or particle flux generated by nonlinear dynamics in plasmas may be reproduced by a linear, though, fractional model. The non-locality is introduced through the integral description of the fractional derivative, and the non-Maxwellian distribution function drives the observed PDFs of densities and heat flux far from Gaussian ${ }^{15}$ as well as shear flow dynamics. ${ }^{16}$ Some of the previous papers on plasma transport have used models including a fractional derivative where the fractional derivative is introduced on phenomenological premises. ${ }^{17}$ In the present work, we introduce the Lévy statistics into the Langevin equation thus yielding a fractional FP description. This approach is similar to that of Refs. 17-19 resulting in a phenomenological description of the non-local effects in plasma turbulence. Using fractional generalizations of the Liouville equation, kinetic descriptions have been developed previously. ${ }^{20-23}$

In investigations of the anomalous character of transport, a useful tool is the non-extensive statistical mechanics which provides distribution functions intermediate to that of Gaussians and Lévy distributions adjustable by a continuous real parameter $q .^{25-27}$ The parameter $q$ describes the degree of non-extensivity in the system. Non-extensive statistical mechanics has a solid theoretical basis for analysing complex systems out of equilibrium where the total entropy is not equal to the sum of the entropies from each subsystem. For systems comprised independent or parts interacting through short-range forces, the Boltzmann-Gibb statistical mechanics is sufficient; however, for systems exhibiting fractal structure or long range correlations, this approach becomes unwarranted. Tsallis statistics is now widely applied, e.g., to solar and space plasmas such as the heliosphere magnetic field and the solar wind. ${ }^{28-30}$

Note that due to the obtained Lévy type distribution functions, higher moments will diverge thus it is of interest to define convergent statistical measures of the underlying process. We will employ the generalized $q$-moments or $q$-expectations as $\left\langle v^{p}\right\rangle_{q}=\int d v F(v)^{q} v^{p} / \int d v F(v)^{q}$. The $q$-expectation can be a convergent moment of the distribution function although the regular moments diverge. This 
also gives us the opportunity to have a convergent pseudoenergy that is always convergent.

The aim of this study is to elucidate on the nonextensive properties of the velocity space statistics and characterization of the fractal process in terms of Tsallis statistics. In Refs. 18 and 19, two limiting cases of the forced FFPE were studied using an expansion in the fractionality parameter $\alpha$ close to two. However, due to the intractability of these models, a minimal model for the FFPE is thus constructed retaining mainly the effects of the fractional operator in order to understand the properties of the FFPE. Furthermore, in order to establish an effective connection to the extended statistical mechanics, we obtain numerical solutions of the PDFs derived from the Tsallis statistic which is in good agreement with those found using the FFPE. ${ }^{32}$ Furthermore, we consider numerical solutions to the Langevin system with Lévy distributed noise and show qualitatively similar results as the analytical work. Moreover, we find that self-organising behavior is present in the system where the ratio of the entropy and energy expectation is decreasing with decreasing fractionality.

The remainder of the paper is organized as in Sec. II the fractional Fokker-Planck equation is introduced, whereas in Sec. III a numerical study of the probability distribution functions obtained is presented. In Sec. IV, the resulting numerical entropies are discussed, while the paper is concluded by results and discussions in Sec. V.

\section{FRACTIONAL FOKKER-PLANCK EQUATION}

The motion of a colloidal particle, i.e., Brownian motion, is described by a stochastic differential equation also known as the Langevin equation. ${ }^{33}$ It is assumed that the influence of the background medium can be split into a dynamical friction and a fluctuating part, $A(t)$, represented by Gaussian white noise. The Gaussian white noise assumption is usually imposed in order to obtain a Maxwellian velocity distribution describing the equilibrium of the Brownian particle. This connection is due to the relation between the Gaussian central limit theorem and classical Boltzmann-Gibbs statistics. ${ }^{34}$ However, the Gaussian central limit theorem is not unique and a generalization was done by Lévy, ${ }^{35}$ Khintchine, ${ }^{34}$ and Seshadri, ${ }^{36}$ by using long tailed distributions.

The underlying physical reasoning is to allow for the non-negligible probability of preferred direction and long jumps, i.e., Lévy flights, which therefore allows for asymmetries and long tails in the equilibrium PDFs, respectively. In the present work, we introduce the Lévy statistics into the Langevin equation thus yielding a fractional FP description. Following the approach used by Barkai ${ }^{37}$ and Moradi, ${ }^{18,19}$ a FFPE with a fractional velocity derivative in the presence of a constant external force is obtained as:

$$
\frac{\partial F}{\partial t}+\mathbf{v} \frac{\partial F}{\partial \mathbf{r}}+\frac{\mathbf{F}}{m} \frac{\partial F}{\partial \mathbf{v}}=\nu \frac{\partial}{\partial \mathbf{v}}(\mathbf{v} F)+D \frac{\partial^{\alpha} F}{\partial|\mathbf{v}|^{\alpha}},
$$

where $0 \leq \alpha \leq 2$. Here, the term $\frac{\partial^{\alpha} F}{\partial|\mathbf{v}|^{\alpha}}$ is the fractional Riesz derivative. The diffusion coefficient, $D$, is related to the damping term $\nu$, according to a generalized Einstein relation $^{37,39}$

$$
D=\frac{2^{\alpha-1} T_{\alpha} \nu}{\Gamma(1+\alpha) m^{\alpha-1}} .
$$

Here, $T_{\alpha}$ is a generalized temperature, and taking force $\mathbf{F}$ to represent the Lorentz force acting on the particles with mass $m$ and $\Gamma(1+\alpha)$ is the Euler gamma function.

In order to analytically investigate the effects of the fractional derivative on the diffusion, we consider the forceless homogeneous one dimensional Fokker-Planck equation of the form

$$
\frac{\partial F}{\partial t}=\nu \frac{\partial}{\partial v}(v F)+D \frac{\partial^{\alpha}}{\partial|v|^{\alpha}} F .
$$

The solution is found by Fourier transforming and treating the fractional derivative in the same manner as in Ref. 38 we find

$$
\frac{\partial \hat{F}}{\partial t}=-\nu k \frac{\partial}{\partial k} \hat{F}-D|k|^{\alpha} \hat{F} .
$$

The stationary PDF is now readily obtained by integration and an inverse Fourier transform

$$
\begin{gathered}
\hat{F}(k)=F_{0} \exp \left(-\frac{D}{\nu \alpha}|k|^{\alpha}\right), \\
F(v)=\frac{F_{0}}{2 \pi} \int_{-\infty}^{\infty} d k \exp \left(-\frac{D}{\nu \alpha}|k|^{\alpha}+i k v\right) .
\end{gathered}
$$

Due to the fractal form of the inverse Fourier transform, analytical solutions of the PDF for the general case are difficult to obtain, except in particular, cases of $\alpha=1.0$ and $\alpha=2$ yielding a Lorentz distribution and a Gaussian distribution, respectively. Note that Eq. (6) is equivalent to what was found in Ref. 23. From a different perspective, for a PDF of a single variable, the Tsallis statistics may be generated by an appropriately constructed Langevin equation of the form

$$
\frac{d v}{d t}=K(v)+\frac{d D(v)}{d v}+\sqrt{2 D(v)} w(t) .
$$

This result was obtained in Ref. 40, where $K(v), D(v)$, and $d D(v) / d v$ are non-stochastic functions of $v$ and $w(t)$ is whitein-time Gaussian noise. The PDF generated by Eq. (7) is

$$
F(v)=\frac{N}{\left(1+\beta(q-1) v^{2}\right)^{1 /(q-1)}} .
$$

Note that $q>1$ and $\beta$ are found from the analytical forms of $D(v)$ and $K(v)$ as well as the $v^{2}$ dependence. Here, $N$ is normalization factor. Furthermore, it is found that $\beta$ is not representative of an inverse temperature of the system due to its non-equilibrium nature. It has been recognized that multifractal models stemming from the Tsallis statistical mechanics may well describe isotropic fluid turbulence at high but finite Reynolds number. ${ }^{24}$ In Sec. III, we will study the solutions to Eq. (6) in more detail. 


\section{NUMERICAL SOLUTIONS TO THE FRACTIONAL FOKKER-PLANCK EQUATION}

The main topic of this paper is to evaluate the statistical properties in terms of Tsallis statistics dependent of the fractional index $(\alpha)$ in Eq. (6). We will start by numerically computing the PDFs with $\alpha$ as our free parameter, and next we will fit the computed PDFs to the proposed generalized analytical Cauchy-Lorentz PDFs found from Tsallis statistical mechanics. Subsequently, in order to statistically evaluate the numerically found PDFs in the fractal model, we will determine the $q$-expectation and the Tsallis non-extensive entropy. Note that the regular statistical moments of the PDFs will not converge unless the PDFs are considered to have a finite compact support. Although the PDFs have been studied before in Refs. 31 and 32, we show the PDFs and fits to the analytically found PDFs for completeness and for reference during the $q$-entropy study. Thus, we will now focus on solving Eq. (6) numerically by computing the inverse Fourier transform and compare the found PDFs to previously derived analytical solutions.

In Figure 1, the numerically found PDFs are shown (log-linearly) for $\alpha=0.25$ (black dashed line), $\alpha=0.50$ (cyan line), $\alpha=0.75$ (yellow line), $\alpha=1.00$ (magenta line), $\alpha=1.25$ (green line), $\alpha=1.50$ (red line), $\alpha=1.75$ (blue line), and $\alpha=2.00$ (black line). Here, in this study, the diffusion coefficient over the dissipation is kept constant $D / \nu=1.0$.

We note that as the parameter $\alpha$ decreases, the normalized fourth moment (Kurtosis $=m_{4} / m_{2}^{2}=$ the ratio of the fourth moment divided by the square of the standard deviation) of the symmetric PDF increases rapidly where PDFs become more and more peaked with elevated tails. Note that the distribution varies smoothly as parameter $\alpha$ is decreased from a Gaussian distribution with $\alpha=2.0$ passing through the Lorentz distribution with $\alpha=1.0$.

In line with the stochastic non-linear analysis presented in Eqs. (7)-(11), it has been shown, in Ref. 31, that using generalized statistical mechanics yielded PDFs that are of Cauchy-Lorentz form

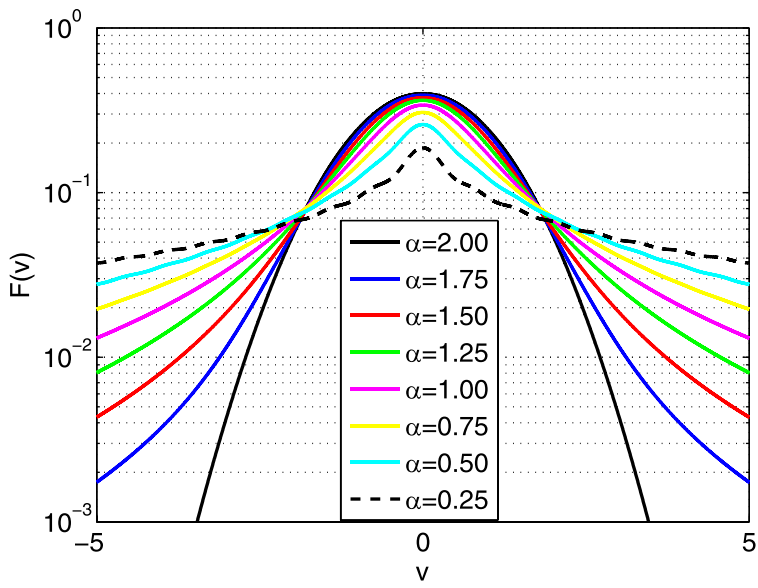

FIG. 1. The $\mathrm{F}(\mathrm{v})$ as a function of the velocity $\mathrm{v}$ for $\alpha=2.00$ (black line), $\alpha=1.75$ (blue line), $\alpha=1.50$ (red line), $\alpha=1.25$ (green line), $\alpha=1.00$ (magenta line), $\alpha=0.75$ (yellow line), $\alpha=0.50$ (cyan line), and $\alpha=0.25$ (black dashed line).

$$
F(v)=\frac{a}{\left(1+b(q-1) v^{2}\right)^{1 /(q-1)}} .
$$

We note that this type of PDF exhibits power law tails that are significantly elevated compared to Gaussian or exponential tails, cf. Eq. (7). It is worthy noting that the precise analytical relation between the fractality index $\alpha$ and the non-extensivity parameter $q$ is not entirely clear. One possibility is the formal relation between the fractality index $\alpha$ and the non-extensivity $q$ proposed by Tsallis et al. ${ }^{31}$ as

$$
\alpha=\frac{3-q}{q-1} .
$$

A second possibility is the following simple relation in Ref. 31:

$$
\alpha=\frac{1}{q-1} .
$$

Taking Gaussian limit in Eq. (11) requires a caution as it cannot reproduce the limit of $\alpha=2.0$ where $q=1$. Interestingly, comparing Eq. (11) there is a direct connection between non-linear dynamics and the fractional FP model. However, Eq. (11) yields a seemingly erroneous scaling of the tail of the PDF. It can easily be shown that $F(v) \propto$ $v^{-(\alpha+1)}$ as $v \rightarrow \infty$ which is only fulfilled by Eq. (10), whereas (11) yields a steeper slope for the tails with $\alpha$. On the other hand, in the limit of small $v$, the exponential factor can be approximated as follows $e^{i k v}=1+i k v-\frac{1}{2} k^{2} v^{2}+\cdots$, keeping only the even powers in the integral due to the symmetry, we find

$F(v) \approx \frac{F_{0}}{\pi} \frac{1}{\alpha}\left[\left(\frac{D}{\alpha \nu}\right)^{-1 / \alpha} \Gamma\left(\frac{1}{\alpha}\right)-\left(\frac{D}{\alpha \nu}\right)^{-3 / \alpha} \Gamma\left(\frac{3}{\alpha}\right) \frac{v^{2}}{2 !}+\cdots\right]$.

Here, $\Gamma$ is the gamma function. Note that for large $\alpha$, the relations from Eqs. (10) and (11) yield similar values and asymptotically approaches 1 . Moreover, the last expansion in Eq. (12) approximates a Gaussian distribution function for small $v$ for both Eqs. (10) and (11).

To further investigate the suitability of Eqs. (10) and (11), in Figure 2, the PDFs are fitted using Eq. (11) with $q=5 / 3$ and utilizing (10) with $q=9 / 5$ for $\alpha=3 / 2$. We find good agreement over several orders of magnitude between the proposed analytically derived PDFs based on Eqs. (11) and (10) and the numerically computed PDFs for the values used. Note that similar agreement is found for all values of $0.25<\alpha<1.5$. We find that using $q=5 / 3$ gives a slightly better fit for small $v$ compared to the higher $q=9 / 5$ value whereas, the tails seem to be off using Eq. (11). Since the tail parts are of great importance, we will thus use the relation in Eq. (10) throughout the rest of the paper.

While we follow the definition that any diffusive process that diverges from the form $\left\langle x^{2}\right\rangle(t) \propto t$ is called anomalous, in most cases we will deal with super-diffusion where $\left\langle x^{2}\right\rangle(t)$ may be divergent. In order to find a useful statistical measure of the super-diffusive or fractal process, we introduce 


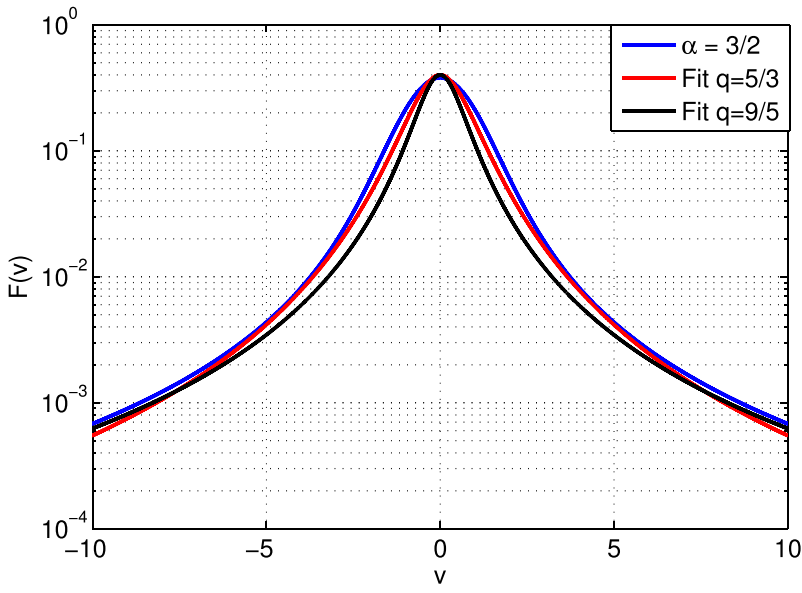

FIG. 2. The PDF as a function of the velocity $v$ for $\alpha=1.5$ (blue line) fitted with $q=5 / 3$ (red line) and $q=9 / 5$ (black line).

$$
\left\langle v^{2}\right\rangle_{q}=\frac{\int_{-\infty}^{\infty} d v(F(v))^{q} v^{2}}{\int_{-\infty}^{\infty} d v(F(v))^{q}}
$$

which we will call the $q$-expectation according to Ref. 32 . Note that, e.g., the exactly solvable case with $\alpha=1.0$, we find that the ordinary expectation diverges; however, as $q$ increases a finite measure is found. Moreover, this gives also the opportunity to define a pseudo-energy in the system as the smallest possible value $q$, where the $q$-expectation converges. Naturally, this reduces to the classical energy for $\alpha=2$.

In principle, all values of $v F(|v|<\infty)$ should be used for the $q$-expectation of $\mathrm{F}(\mathrm{v})$. However, for numerical tractability, we have used a PDF with finite support $F(v)_{\text {num }}=F(v)$ for $|v|<10$ and zero everywhere else. Different support ranges have been tested where extending the range $|v|<15$ makes only minor changes.

Figure 3 shows the $q$-expectation as a function of $q$, treating $q$ as a free parameter for $\alpha=0.5$ (magenta line), $\alpha=1.0$ (blue line), $\alpha=1.5$ (red line), and $\alpha=2.0$ (black line). We find that just as expected, the $q$-expectation falls off with $q$; however, results corresponding to smaller $\alpha$ (more intermittent) fall off faster than the $q$-expectation of

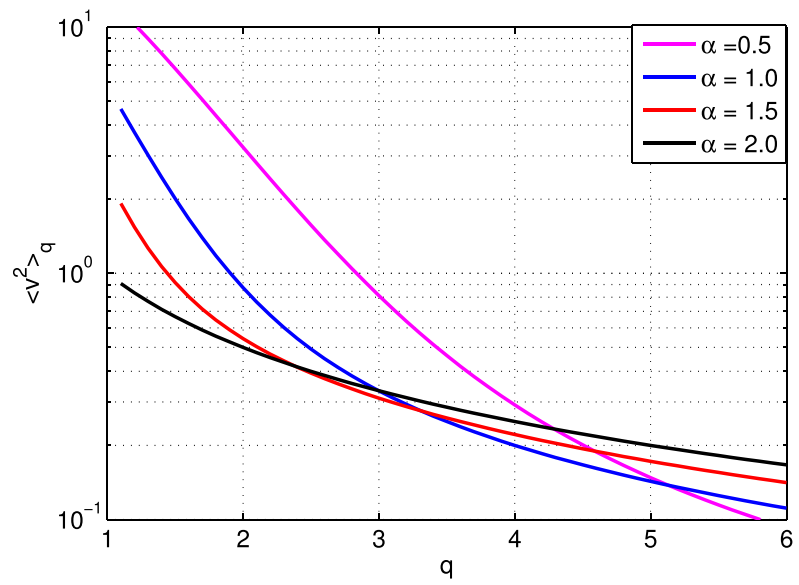

FIG. 3. The normalized $q$-expectation as a function of $q$ for $\alpha=0.5$ (magenta line), $\alpha=1.0$ (blue line), $\alpha=1.5$ (red line), and $\alpha=2.0$ (black line).

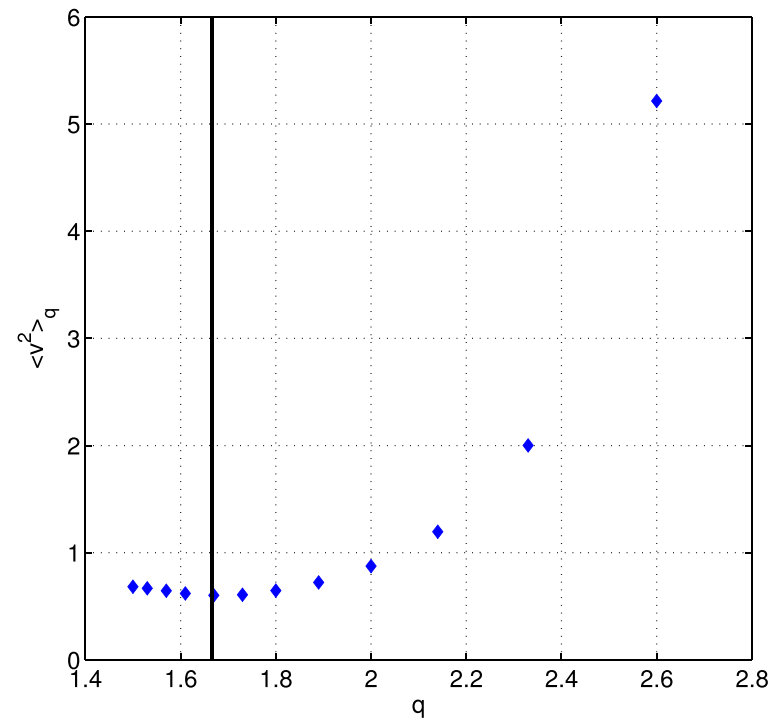

FIG. 4. The normalized $q$-expectation (blue stars) as a function of $q$ using Eq. (10) as a relation between the non-extensivity and the fractality with a vertical line at $q=5 / 3$.

the Gaussian process. In general, there exists a smallest value for $q$, where the $q$-expectation is finite. In the case of $\alpha=2.0$, it converges for all $q$ and we find that the PDF is a Gaussian with a variance $\sigma^{2}=1.0$.

In Figure 4, we have used the relation in Eq. (10) to determine the appropriate values for the different $\alpha$-stable processes of the PDFs determined in Figure 1, showing a scan from $q \in(1.5,2.6)$ with a vertical line at $q=5 / 3$. We find that the $q$-expectation has a minimum at $q=5 / 3$, which is equivalent to value of $\alpha=2.0$. This further confirms that Eq. (10) is a suitable choice and also that the $q$-expectation is a useful measure.

Furthermore, in Figure 5, the 1-expectation or energy is shown as a function (numerical result in black dots and a fit in red diamonds) of $\alpha$ utilizing the finite support of the PDFs shown in Figure 1. It is found that the 1-expectation decreases exponentially with increasing $\alpha$. Here, we also note that the energy is convergent for all $\alpha$ due to the finite support of the PDFs. The main motivation for defining the $q$-expectation or tempered pseudo-energy is the sharp

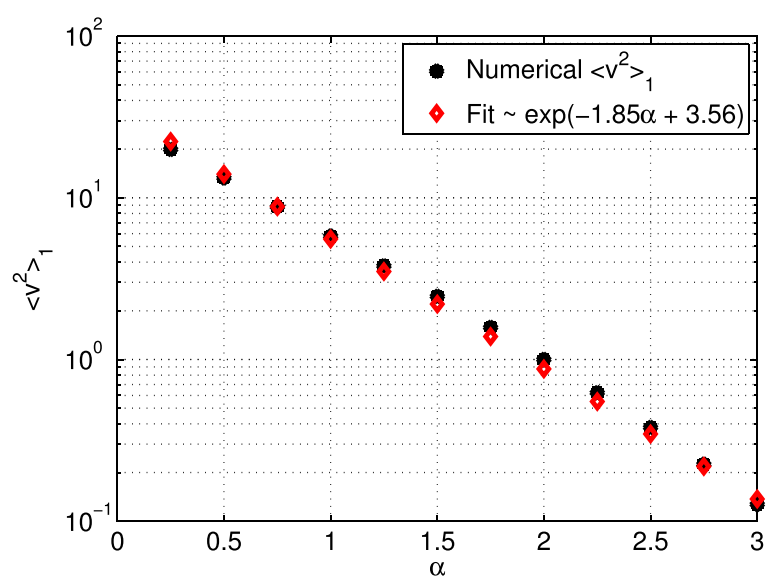

FIG. 5. The 1-expectation as a function of $\alpha$ for the numerically bounded PDFs with the numerical result in black dots and a fit in red diamonds. 
increase in the second moment for small $\alpha$, where the expectation value is diverging due to the long tails of the PDFs.

\section{ENTROPY}

In thermodynamics, a measure on the number of ways a system can be arranged is denoted entropy. In terms of generalized statistical mechanics, $q$-entropy or Tsallis entropy can be introduced as

$$
S_{q}=\frac{1-\int d v(F(v))^{q}}{q-1} .
$$

Note that for Gaussian statistics, the $q$-entropy is reduced (by L'Hospital's rule) to the conventional Boltzmann-Gibbs entropy $S=-\int d v \log (F(v)) F(v)$. Moreover, an important distinct property of the Tsallis generalized entropy is its nonextensivity, i.e., for two systems $A$ and $B$, the total entropy is not the sum of the entropies of the individual systems, $S_{q}(A+B) \neq S_{q}(A)+S_{q}(B)$. We will now use the Tsallis entropy to investigate the importance of fractal structure in velocity space and we will contrast the resulting generalized entropies to the standard Boltzmann-Gibbs entropy.

In Figure 6, the dependence of the $q$-entropy as a function of $q$ for $\alpha=0.5$ (magenta line), $\alpha=1.0$ (blue line), $\alpha=1.5$ (red line), and $\alpha=2.0$ (black line) with $q$ as a free parameter is displayed. The $q$-entropy is rapidly decreasing with increasing $q$, mainly due to the fact that the entropy is dependent on the $q$-th power of the PDF. The scaling of the $q$-entropy of $q$ using relation (10) is displayed in Figure 7 with a vertical line at $q=5 / 3$. Using the appropriate relation between $\alpha$ and $q$ shows that a maximum in the entropy is found for Gaussian statistics. For comparison, the Boltzmann-Gibbs entropy is shown in Figure 8 as a function of $q$ using the relation between $\alpha$ and $q$ as in Eq. (10). We find that the entropy is monotonously increasing with increasing $q$. This is an indication that the Tsallis entropy is viable measure identifying the process with highest entropy as the Gaussian process. As an interesting measure of the dynamics and the importance of fractionality in the dynamics

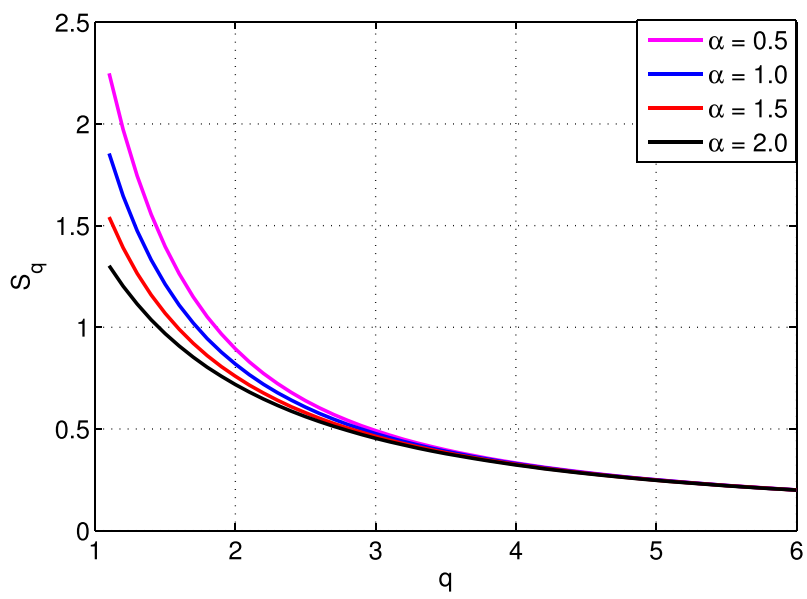

FIG. 6. The $q$-entropy as a function of $q$ for PDFs with $\alpha=0.5$ (magenta line), $\alpha=1.0$ (blue line), $\alpha=1.5$ (red line), and $\alpha=2.0$ (black line).

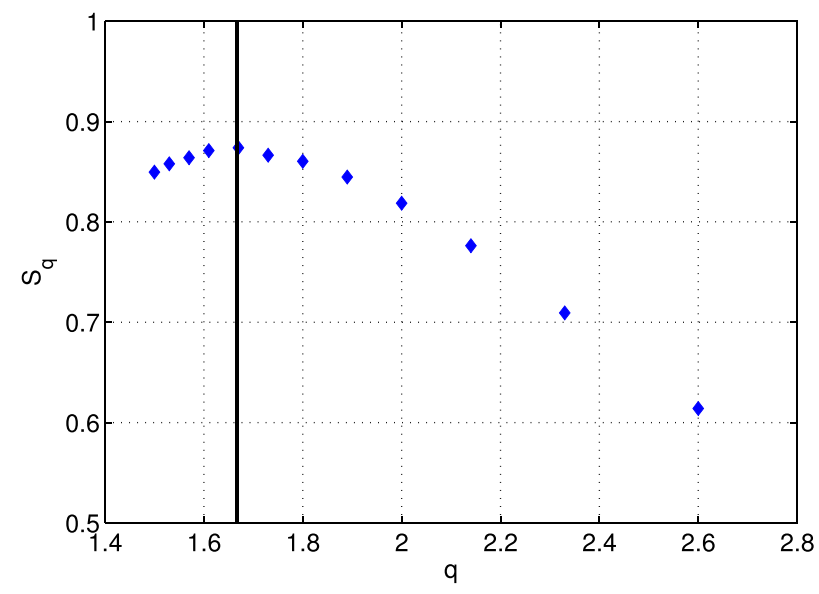

FIG. 7. The $q$-entropy as a function of $q$ using Eq. (10) as a relation between the non-extensivity and the fractality.

are to normalize the entropy with the energy, we will now discuss this quantity.

The $q$-entropy normalized with the $q$-expectation as a function of $q$ is displayed in Figure 9 for $\alpha=1 / 2$ (magenta rings), $\alpha=1.0$ (blue diamonds), $\alpha=3 / 2$ (red triangles), and $\alpha=2.0$ (black stars). The normalized $q$-entropy is rapidly increasing with increasing $q$ mainly due to the rapid decrease of the $q$-expectation $\left(\left\langle v^{2}\right\rangle_{q}\right)$ with increasing $q$. This indicates that in a statistical mechanics sense, the normalized generalized entropy is increasing with increasing $q$ as a process in velocity space in the sub-diffusive domain, whereas in the range of small $\alpha$ the high-velocity, small likelihood events are more dominant. In the sub-diffusive regime, the small amplitude events are dominant. In Figure 10, the $q$-entropy normalized to the $q$ as a function of $q$ determined by the relation 10 is displayed. We find that the maximum is found at the same $q=5 / 3$ values as the vertical line. Furthermore, in contrast in Figure 11, the Boltzmann-Gibbs entropy normalized to the energy (black dots) and the $q$-expectation (blue stars) as a function of $q$ with a vertical line at $q=5 / 3$ is shown. Here, the $q$ values are determined by Eq. (10). The normalized Boltzmann-Gibb entropy increases as a function of $q$ when normalized to the regular energy due to the

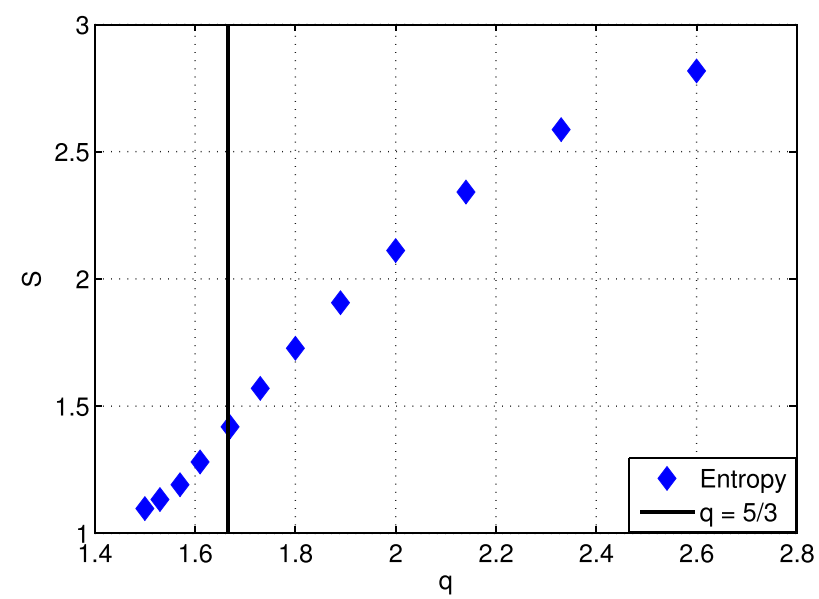

FIG. 8. The Boltzmann-Gibb entropy as a function of $q$ with a vertical line $q=5 / 3$. 


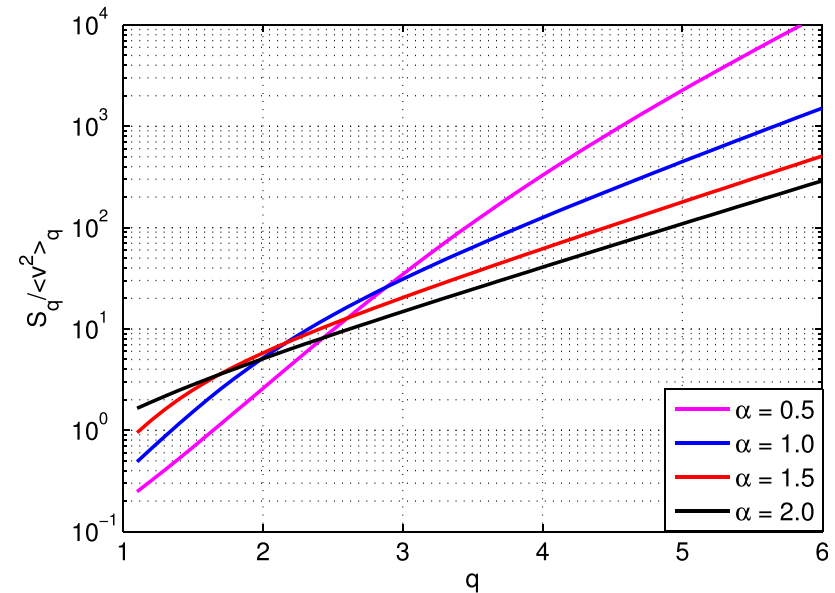

FIG. 9. The $q$-entropy normalized to the $q$-expectation $\left\langle v^{2}\right\rangle_{q}$ as a function of $q$ for PDFs with $\alpha=0.5$ (magenta line), $\alpha=1.0$ (blue line), $\alpha=1.5$ (red line), and $\alpha=2.0$ (black line).

influence of the strong tails. Also in the normalized description, the $q$-entropy can identify the Gaussian process as the process with highest normalized entropy. However, the entropy normalized to the $q$-expectation is first increasing for small $q$ in the sub-diffusive region and then decreases for larger $q$ in the super-diffusive region. The dynamics of the FFPE system is dependent on the level of diffusion in comparison to the collisional damping in terms of the ratio of the two free parameters $D / \nu$, cf. Eq. (6). Allowing for a controlled variation of the free parameter $D / \nu$ is an important aspect on elucidating on the complex fractional dynamics. Note that the width of the PDFs is significantly dependent on this factor, making the normalization with $\left\langle v^{2}\right\rangle$ and $\left\langle v^{2}\right\rangle_{q}$ impossible. In Figures 12 and 13, the q-entropy and Boltzmann-Gibbs entropy are shown with $D / \nu$ as a parameter, respectively. In this study, the discrete values of $D / \nu$ are chosen to be in the range of $\left\{10^{-2}, 10^{-1}, 1,10^{1}, 10^{2}\right\}$. In Figure 12, it is found that the q-entropy is decreasing with $q$ for small $D / \nu$ (collision dominated), whereas the opposite or a flat profile is found for large $D / \nu$ (diffusion dominated). Furthermore, in Figure 13, the Boltzmann-Gibbs entropy is presented where the entropy is increasing with increasing $q$,

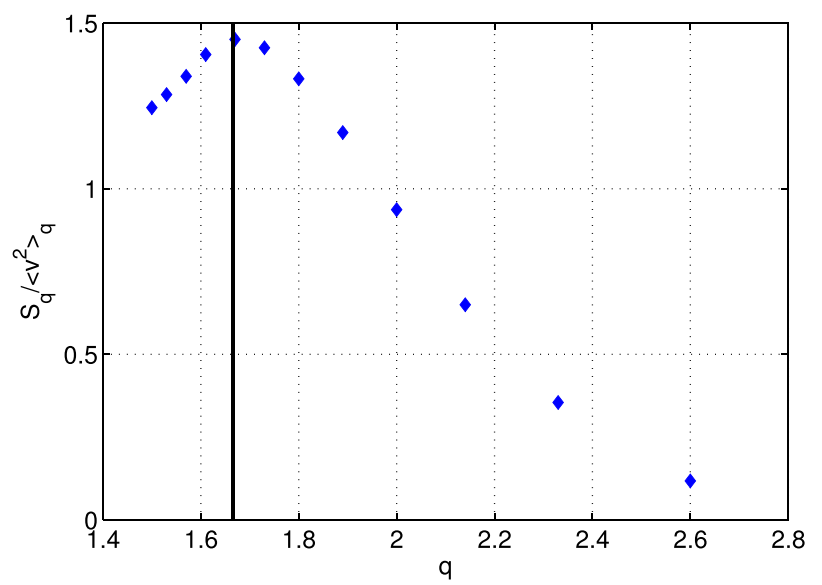

FIG. 10. The $q$-entropy normalized to the $q$-expectation $\left\langle v^{2}\right\rangle_{q}$ as a function of $q$ using Eq. (10) as a relation between the non-extensivity and the fractality with a vertical line at $q=5 / 3$.

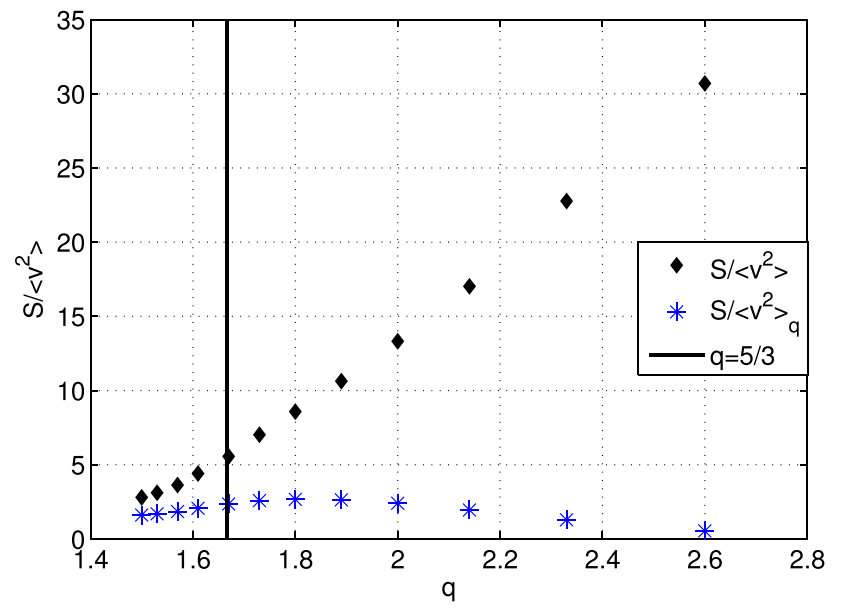

FIG. 11. The Boltzmann-Gibbs entropy normalized to the $q$-expectation $\left\langle v^{2}\right\rangle_{q}$ and the normal variance as a function of $q$ using Eq. (10) as a relation between the non-extensivity and fractality with a vertical line at $q=5 / 3$.

in particular, in the diffusion dominated regime. In the above, it is indicated that as the fractality index increases (smaller q), the entropy decreases indicating a selforganising behavior in velocity space with long-range correlations. The number of possible microscopical realizations decreases fast.

As a comparison to the analytical work, we numerically study the influence of Lévy stable processes as a noise source in the Langevin equation of motion

$$
\begin{gathered}
\frac{d x}{d t}=v, \\
\frac{d \nu}{d t}=-\nu v+\zeta .
\end{gathered}
$$

Here, the variables are normalized in the numerical scheme as $\tau=\nu t$ and $\zeta$ is the Lévy stochastic forcing. In the simulations, ${ }^{39}$ we follow 100000 particles until the system comes to a quasi-steady state. The numerical PDFs are determined by using 200 bins and we consider variations of the fractionality between $\alpha \in\{1.1, \ldots, 2.0\}$. Note that also here the PDFs are found to fall off as $F(v) \sim v^{-\alpha-1}$. The resulting Boltzmann-Gibbs and Tsallis entropies are given in Figure

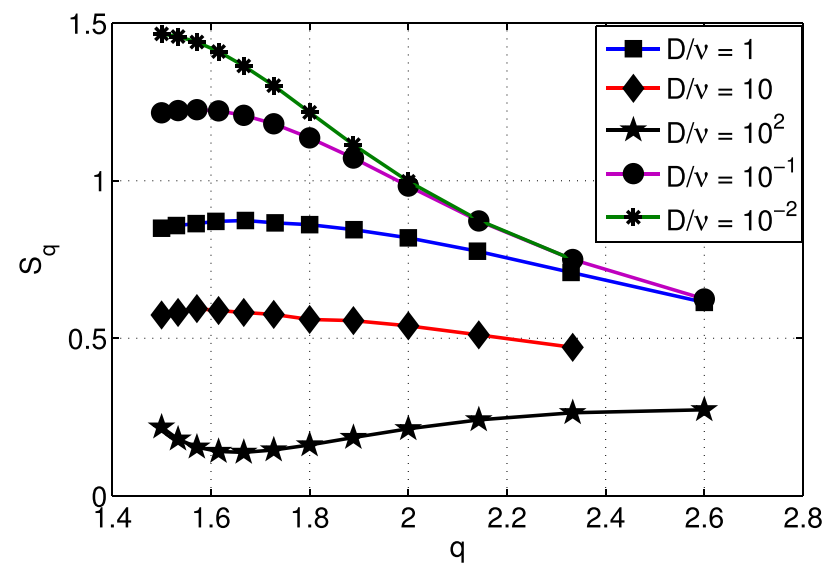

FIG. 12. The q-entropy as a function of $q$ using Eq. (10) as a relation between the non-extensivity. 


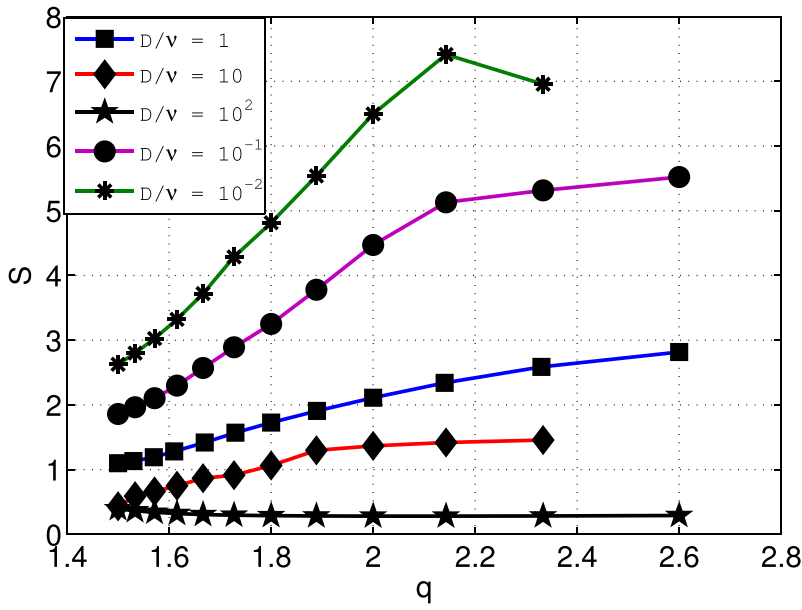

FIG. 13. The Boltzmann-Gibbs entropy as a function of $q$ using Eq. (10) as a relation between the non-extensivity and fractality.

14. Contrasting the results with those shown in Figures 7 and 8 , it is found that the Boltzmann-Gibb entropy is increasing with $q$ and that the Tsallis entropy is almost flat in the current range and finally seems to decrease for larger $q$. This is in good qualitative agreement with the analytical model; however, there are some quantitative differences. Note that the range $q$ is smaller compared to the analytical work.

\section{RESULTS AND DISCUSSION}

Non-linear processes with non-Gaussian character have attracted significant attention during recent years calling for an efficient model describing such dynamics. In this paper, we have investigated one prominent candidate capturing the main features in the dynamics, namely, the Fractional extended Fokker-Planck Equation (FFPE). The FFPE is obtained by modifying the velocity derivative to a fractional differential operator allowing for non-local effects in velocity space. The underlying physical reasoning for using the FFPE is to allow for the non-negligible probability of direction preference and long jumps, i.e., Lévy flights, which therefore allows for asymmetries and long tails in the equilibrium PDFs, respectively.

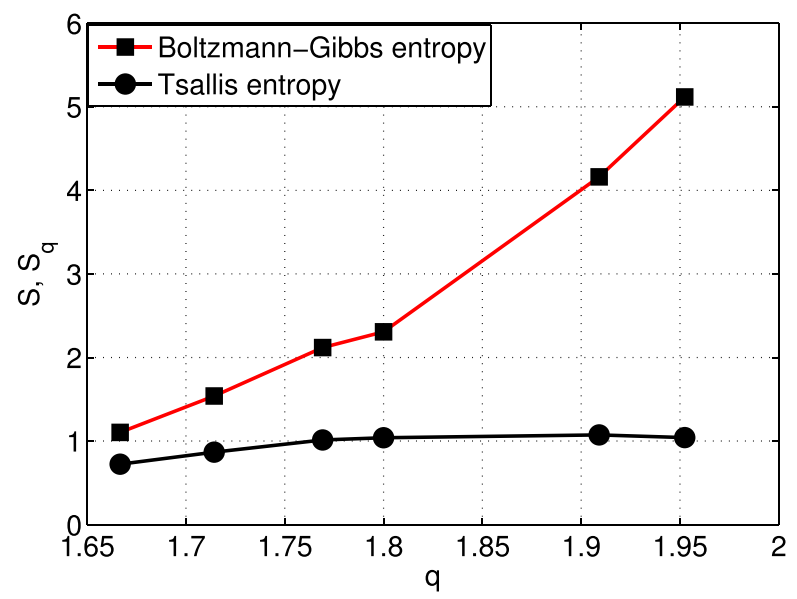

FIG. 14. The numerically found Boltzmann-Gibbs and Tsallis entropies as a function of $q$ using Eq. (10) as a relation between the non-extensivity and fractality.
The aim of this study was to shed light on the nonextensive properties of the velocity space statistics and characterization of the fractal processes of the FFPE in terms of Tsallis statistics. The non-extensive statistical mechanics of Tsallis provides velocity space distribution functions intermediate to that of Gaussians and Lévy distributions adjustable by a continuous real parameter $q$ which seems to be suitable for comparing with the distribution found in FFPE. The parameter $q$ describes the degree of non-extensivity in the system. Non-extensive statistical mechanics has a solid theoretical basis for analysing complex systems out of equilibrium. For systems comprised independent or parts interacting through short-range forces, the Boltzmann-Gibb statistical mechanics is sufficient; however, for systems exhibiting fractal structure or long range correlations, this approach becomes unwarranted.

In this work, we have utilized generalized $q$-moments or $q$-expectations as $\left\langle v^{p}\right\rangle_{q}=\int d v F(v)^{q} v^{p} / \int d v F(v)^{q}$ due to the obtained Lévy type character of the distributions, higher moments may diverge. The $q$-expectation results in convergent moment although the regular moments diverge. This also permits us to define a pseudo-energy that is always convergent. We show that the PDFs derived from the Tsallis statistic are in good agreement with those found using the FFPE. Moreover, we find that self-organising behavior is present in the system where the ratio of the entropy and energy expectation is decreasing with decreasing fractionality or increasing $\alpha$.

Finally, it seems that a FFPE is a viable candidate for explaining certain non-linear features ubiquitous to anomalous plasma transport as well as for other physical processes. Note that in Ref. 24, a relation between Tsallis statistical mechanics and Navier-Stokes turbulence was established. A direct numerical comparison between the Langevin approach and the FFPE using Tsallis statistical mechanics is a possible topic for the future work.

\section{ACKNOWLEDGMENTS}

The authors are grateful to the participants of Festival de théorie 2013, organized by the C.E.A of France, for many valuable discussions.

${ }^{1}$ M. Schlesinger, G. M. Zaslavsky, and J. Klafter, Nature 363, 31 (1993).

${ }^{2}$ I. M. Sokolov, J. Klafter, and A. Blumen, Phys. Today 55(11), 48 (2002).

${ }^{3}$ J. Klafter and I. M. Sokolov, Phys. World 08, 29 (2005).

${ }^{4}$ R. Metzler and J. Klafter, Phys. Rep. 339, 1 (2000).

${ }^{5}$ R. Metzler and J. Klafter, J. Phys. A: Math. Gen. 37, R161 (2004).

${ }^{6}$ B. B. Mandelbrot, The Fractal Geometry of Nature (W. H. Freeman and Company, San Francisco, 1982).

${ }^{7}$ J. A. Krommes, Phys. Rep. 360, 1-352 (2002).

${ }^{8}$ E. W. Montroll and H. Scher, J. Stat. Phys. 9, 101 (1973).

${ }^{9}$ S. C. Kou and X. Sunney Xie, Phys. Rev. Lett. 93, 180603 (2004).

${ }^{10}$ B. A. Carreras, C. Hidalgo, E. Sanchez, M. A. Pedrosa, R. Balbin, I. Garcia-Cortes, B. van Milligen, D. E. Newman, and V. E. Lynch, Phys. Plasmas 3, 2664 (1996).

${ }^{11}$ B. A. Carreras, B. Ph. van Milligen, C. Hidalgo, R. Balbin, E. Sanchez, I. Garcia-Cortes, M. A. Pedrosa, J. Bleuel, and E. Endler, Phys. Rev. Lett. 83, 3653 (1999).

${ }^{12}$ B. Ph. van Milligen, R. Sanchez, B. A. Carreras, V. E. Lynch, B. LaBombard, M. A. Pedrosa, C. Hidalgo, B. Goncalves, R. Balbin, and W7-As Team, Phys. Plasmas 12, 052507 (2005). 
${ }^{13}$ R. Sanchez, D. E. Newman, J.-N. Leboeuf, V. K. Decyk, and B. A. Carreras, Phys. Rev. Lett. 101, 205002 (2008).

${ }^{14}$ D. del-Castillo-Negrete, B. A. Carreras, and V. E. Lynch, Phys. Rev. Lett. 94, 065003 (2005).

${ }^{15}$ J. Anderson and P. Xanthopoulos, Phys. Plasmas 17, 110702 (2010).

${ }^{16}$ E. Kim, H. Liu, and J. Anderson, Phys. Plasmas 16, 052304 (2009).

${ }^{17}$ R. Sanchez, B. A. Carreras, D. E. Newman, V. E. Lynch, and B. Ph. van Milligen, Phys. Rev. E 74, 016305 (2006).

${ }^{18}$ S. Moradi, J. Anderson, and B. Weyssow, Phys. Plasmas 18, 062106 (2011).

${ }^{19} \mathrm{~S}$. Moradi and J. Anderson, Phys. Plasmas 19, 082307 (2012).

${ }^{20}$ G. M. Zaslavsky, Hamiltonian Chaos and Fractional Dynamics (Oxford University Press, Oxford, 2005).

${ }^{21}$ G. M. Zaslavsky, Phys. Rep. 371, 461 (2002).

${ }^{22}$ V. E. Tarasov, J. Phys.: Conf. Ser. 7, 17 (2005).

${ }^{23}$ V. E. Tarasov, Chaos 16, 033108 (2006).

${ }^{24}$ T. Gotoh and R. Kraichnan, Physica D 193, 231 (2004).

${ }^{25}$ C. Tsallis, A. M. C. de Souza, and R. Maynard, Lect. Notes Phys. 450, 269 (1995).

${ }^{26}$ C. Tsallis and D. J. Bukman, Phys. Rev. E 54, R2197 (1996).

${ }^{27}$ C. Tsallis, R. S. Mendes, and A. R. Plastino, Physica A 261, 534 (1998).
${ }^{28}$ G. Balasis, I. A. Daglis, A. Anastasiadis, C. Papadimitriou, M. Mandea, and K. Eftaxias, Physica A 390, 341 (2011).

${ }^{29}$ G. P. Pavlos, L. P. Karkatsanis, M. N. Xenakis, D. Sarafopoulos, and E. G. Pavlos, Physica A 391, 3069 (2012).

${ }^{30}$ G. P. Pavlos, L. P. Karkatsanis, and M. N. Xenakis, Physica A 391, 6287 (2012).

${ }^{31}$ C. Tsallis, S. V. F. Levy, A. M. C. Souza, and R. Maynard, Phys. Rev. Lett. 75, 3589 (1995).

${ }^{32}$ D. Prato and C. Tsallis, Phys. Rev. E 60, 2398 (1999).

${ }^{33}$ S. Chandrasekhar, Rev. Mod. Phys. 21, 383 (1949).

${ }^{34}$ A. Y. Khintchine, The Mathematical Foundation of Statistical Mechanics (Dover, New York, 1948).

${ }^{35} \mathrm{P}$. Lévy, Theorie del'Addition des Variables (Gauthier-Villiers, Paris, 1937).

${ }^{36}$ B. J. West and V. Seshadri, Physica A 113, 203 (1982).

${ }^{37}$ E. Barkai, Phys. Rev. E 68, 055104(R) (2003).

${ }^{38}$ A. I. Saichev and G. M. Zaslavsky, Chaos 7, 753 (1997).

${ }^{39}$ S. Moradi, D. del-Castillo-Negrete, and J. Anderson, "Non-local transport driven by non-Gaussian (Lévy) plasma fluctuations" (unpublished).

${ }^{40}$ E. Lutz, Phys. Rev. A 67, 051402 (2003). 